\title{
El clima institucional y su incidencia en la deserción escolar en sétimo año
}

\section{Roxy Calderón Mora}

Máster en la Enseñanza del Inglés, Universidad Nacional. Máster en Planificación Curricular, Universidad de Costa Rica. Labora como Asesora Nacional de inglés en el Ministerio de Educación Pública y como encargada de Trabajos Finales de Graduación en la Universidad Estatal a Distancia. Dirección electrónica: rcalderon@uned.ac.cr

Recibido: 29 de Agosto 2012 • Aceptado: 12 de Abril 2013

\section{RESUMEN}

La deserción estudiantil, en el sétimo año, plantea al sistema educativo costarricense el reto de buscar nuevas alternativas que impacten en la permanencia de los estudiantes. Este artículo se desprende de un estudio investigativo realizado en el segundo semestre del 2011, consideró entre sus fuentes primarias al director, al orientador, al profesor guía, profesores de asignaturas regulares del currículum y estudiantes de sétimo año en tres colegios de la Dirección Regional de Enseñanza de Cartago. Dicho estudio analiza el clima institucional como un factor de riesgo en la deserción estudiantil, planteándolo como un constructo complejo y dinámico que debe ser abordado en el centro educativo. Variables como la cultura institucional, estructura organizativa, la gestión administrativa, las características profesionales, la convivencia entre los integrantes de la comunidad educativa, la mediación pedagógica y la comunicación constituyen un proceso complejo que debe ser analizado a lo interno de la institución con el propósito de visualizar los desafíos pedagógicos y administrativos en forma integral.

Palabras claves: Deserción escolar, clima institucional, gestión administrativa.

\section{ABSTRACT}

To reduce the amount of students dropping out of school in $7^{\text {th }}$ grade is a challenge for the costarican educational system. This situation prompts authorities to look for different alternatives motivating students to keep on in the formal education. The article reports a descriptive research conducted in the second semester of 2011. Among the sample population the researchers considered the principal, the counselor, the guide teacher, regular teachers and seventh grade students from three chosen high schools, located in the Dirección Regional de Enseñanza de Cartago. The study analyzed the school environment as a risky factor to drop out of school in $7^{\text {th }}$ grade. Factors that influence school environment are considered complex and dynamic; some of the variables are: institutional culture, organizational structure, school management, teachers' profile, sharing among the school community, teaching and learning process and communication. The researcher goal is to provide evidence sustaining that pedagogical and administrative aspects must be approached from the inside of each school, going beyond and see each school as a whole that must fulfill students' needs and interests from different axes and perspectives.

Key words: Dropping out of school, school atmosphere, school management. 


\section{Presentación}

El presente artículo sintetiza los hallazgos de la investigación que lleva por título "Clima institucional y su incidencia en la deserción escolar: una experiencia en sétimo año de los colegios públicos de la Dirección Regional de Cartago: Francisca Carrasco, Daniel Oduber Quirós y Danilo Jiménez Veiga", realizado en el año 2011, para optar por el grado de licenciatura en Administración Educativa. La autora propone considerar seriamente el impacto del clima institucional como un factor de riesgo en la deserción escolar y plantea el clima institucional como un constructo complejo y dinámico que debe ser abordado a lo interno de cada centro educativo.

\section{Introducción}

Uno de los principales retos del sistema educativo costarricense es influir positivamente en las decisiones de la sociedad, esto a pesar de los múltiples factores económicos, sociales, políti$\cos$, entre otros, que intervienen en la construcción social. La Constitución Política de Costa Rica (1979) establece, en su artículo $\mathrm{N}^{\circ} 78$, que la educación preescolar y la general básica (hasta noveno año) son obligatorias y costeadas por la nación. Este artículo obliga al gobierno, instituciones educativas y padres de familia a garantizar las condiciones y el acceso para que todos los niños y adolescentes concluyan sus estudios y obtengan el título de Enseñanza General Básica.

Indudablemente la educación formal cumple una función primordial como proceso transformador que permite el desarrollo humano, económico, político y social de un país; de ahí que las diferentes administraciones se han preocupado por realizar esfuerzos que garanticen la calidad en el sector educativo. Entre las acciones más recientes se destaca la de la administración Chinchilla (2010-2014), que ha establecido como una de sus Diez Líneas Estratégicas de la Educación, "El centro educativo de calidad como un eje de la educación costarricense". Esto implica, según el Consejo Superior de Educación: “... otorgar al centro educativo de calidad la condición de eje central de la política educativa nacional y pilar esencial de la operación del sistema; es la ideafuerza que propiciará la construcción de centros educativos con una identidad institucional más rica, con mayor autonomía relativa, capacidad de decisión y con una participación más amplia de la comunidad educativa" (Ministerio de Educación Pública, 2010). Para concretar ese eje, el gobierno estableció como acción el empoderamiento de los directores de los centros educativos, junto al nuevo papel de los supervisores, para contribuir a fomentar la identidad y la calidad de cada centro educativo.

Como acción para apoyar la labor del centro educativo, el actual Ministro de Educación planteó reformas en la educación secundaria, implementado un plan llamado Educación en Ética, Estética y Ciudadanía, que establece la implementación de nuevos programas de estudio y una mediación pedagógica atractiva en Educación Cívica, Educación Musical, Artes Pláticas y Educación Física. Adicionalmente, dichas gestiones han fortalecido el Programa Avancemos que brinda un apoyo económico sostenido a los estudiantes que provienen de familias con pocos ingresos económicos.

A pesar de estos y otros esfuerzos realizados en el nivel central, la deserción estudiantil plantea al sistema educativo el reto de buscar nuevas alternativas que impacten en la disminución del abandono de las aulas en el sétimo año. Constantemente en los medios de comunicación se informa sobre la deserción escolar como un factor importante de riesgo social. El periódico La Nación, del día 15 de marzo del año 2011, presenta una entrevista en la que el señor ministro de Educación, Leonardo Garnier, indica que "[...] es imposible que la deserción se reduzca de la noche a la mañana; acá cambiamos la tendencia y logramos mantenerla a la baja" (La Nación, 2011, p. 5A).

Finalmente, reducir el abandono escolar en gran escala debería ser una meta a corto plazo; no obstante, las variables son innumerables por lo que la mayoría de las propuestas gubernamentales se han orientado a solventar la situación 
atacando la deserción desde los niveles centrales, sin ser en muchos de los casos efectivas. Ante esta situación, se plantea como un problema de investigación, ¿cuál es la incidencia del clima institucional en la deserción escolar de los estudiantes de sétimo año de los colegios públicos Francisca Carrasco, Daniel Oduber Quirós y Danilo Jiménez Veiga de la Dirección Regional de Cartago? El estudio de la temática permitió indagar y analizar sobre la incidencia del clima institucional como un factor de riesgo en la deserción escolar y ofrecer recomendaciones para que el tema se aborde valorando la situación como un problema educativo en los centros de enseñanza.

\section{Referente teórico}

El planteamiento teórico de la investigación se hace a partir de conceptos que constituyen un marco de referencia para interpretar los resultados del estudio. La discusión teórica aborda perspectivas que involucran a la comunidad educativa desde tres grandes temas, y que constituyen las categorías de análisis; a saber: cultura institucional, mediación pedagógica y gestión administrativa.

\section{Cultura Institucional}

La cultura institucional involucra la identidad de las personas que laboran en el centro educativo, y se construye a partir del tipo de interacciones que se despliegan en su interior. El comportamiento de las personas dentro de su ámbito laboral, las funciones que desempeñan y sus interrelaciones personales son elementos que garantizan la efectividad de la organización; en este caso, en la comunidad educativa. La interacción entre sus miembros y con el medio circundante genera una convivencia organizacional y una dinámica de grupo mediatizada por el contexto donde se da dicho intercambio. Los individuos que conforman una institución son la base para que esta logre el máximo desempeño.

Por lo tanto, es de suma importancia conocer y potenciar las características, personalidad, motivación individual, habilidades, valores, el desempeño de eso individuos y los incentivos intrínsecos y extrínsecos que pueden anhelar individual y colectivamente. Gibson y otros (2001) afirman que el comportamiento organizacional es

El campo de estudios que utiliza la teoría, los métodos y los principios de varias disciplinas para aprender sobre las percepciones de los individuos, sus valores, capacidades de aprendizaje y acciones mientras trabajan en grupos dentro de la organización; analizando el efecto del entorno externo sobre la organización y sus recursos humanos, sus misiones, objetivos y estrategias (p. 5).

En esta convivencia humana surge la urgente necesidad de un clima agradable, de entusiasmo e interés para los miembros de la microcomunidad institucional. Adicionalmente, las personas suelen motivarse cuando encuentran un espacio de construcción colectiva impulsada por metas comunes en su lugar de trabajo. Un factor para considerar en este ámbito de acción es la comunicación asertiva; entendida como espacios de expresión oral y escrita que permiten influir de manera favorable y positiva en los asuntos cotidianos y en el comportamiento organizacional. En esencia, una comunicación eficaz es la respuesta a un compromiso de colaboración entre los miembros de la comunidad educativa, lo que resulta esencial para promover procesos de participación, cambio y mejora en los centros educativos.

\section{Mediación Pedagógica}

A partir de la cultura institucional que se genera en los centros educativos, la mediación pedagógica constituye una labor didáctica en el aula y de convicción personal indispensable para el desarrollo de las destrezas, habilidades y actitudes de los educandos quienes deben integrase a una sociedad cada vez más competitiva. Parafraseando a Jabif (2009), en este proceso pedagógico se requiere que los docentes tengan competencias y habilidades que los califiquen para el ejercicio de una profesión que requiere de ciencia, de arte y de praxis. Por su parte, el estudiante es el centro del proceso educativo; todas las acciones y esfuerzos que se desarrollen en la institución deben estar enfocadas en él como un ser humano integral. 
En este proceso, además de los aprendizajes cognitivos, existen habilidades sociales que deben ser desarrolladas.

\section{Gestión Administrativa}

La comunidad educativa es liderada por el director quien se encarga de orientar las acciones institucionales y por ende ser un actor fundamental en la construcción de la identidad del centro educativo. En esta línea de pensamiento Letjman (1996) considera que el director "influye para lograr que un equipo de gente trabaje dentro de cierto estilo, con un objetivo común, con compromiso, responsabilidad y gusto por lo que hace...." (p.84). El recurso humano de la institución, requiere que el director como líder cree condiciones de trabajo que impliquen un compromiso y un desafío para todo el recurso humano. De ahí que sea indispensable que el líder posea cualidades y habilidades de tres tipos: técnicas, humanas y conceptuales. La organización debe adaptarse y actuar conforme a las exigencias del entorno. El buen liderazgo escolar permite transformar los sentimientos, actitudes y opiniones de los miembros de la comunidad educativa, así como sus prácticas para mejorar la cultura de la escuela.

Diferentes autores plantean estilos de administración, los cuales, en la práctica, se combinan por diversas razones y circunstancias. No obstante, el predominio de un estilo participativo en la gestión administrativa propicia espacios de reflexión en un marco en el que la enseñanza, el aprendizaje y la experiencia organizativa, generan información sobre alternativas para tomar decisiones e influir positivamente en el clima institucional.

La planificación, como una fase esencial del proceso de la gestión administrativa de una institución, pone en perspectiva el propósito que se pretende lograr y la forma como se realizará; tiene un carácter instrumental, pues permite orientar el quehacer de los recursos humanos, financieros y físicos mediante un conjunto articulado de técnicas, instrumentos y herramientas específicas para la consecución de las metas planteadas. En este sentido, corresponde a los diferentes representantes de la comunidad educativa brindar los insumos para que el plan estratégico institucional se convierta en instrumento de planificación y de ejecución de acciones en una dimensión no tradicionalista. Se debe plasmar en dicho plan las necesidades y demandas de la comunidad educativa mediante un proceso de reflexión, análisis y cuestionamientos de las propuestas.

\section{Metodología}

\section{Enfoque metodológico}

El estudio sobre el que se sustenta este artículo permitió conocer mediante un abordaje descriptivo, las relaciones que existen entre el clima escolar y la deserción de estudiantes de sétimo año. Según Hernández, Fernández y Baptista (2010) la investigación descriptiva "busca especificar propiedades, características y rasgos importantes de cualquier fenómeno que analice" (p.80). Este se plantea desde un enfoque investigativo mixto, que permite acercarse al objeto de estudio y profundizar en él desde diversas perspectivas. Hernández y otros (2010) consideran que los métodos mixtos "representan un conjunto de procesos sistemáticos, empíricos y críticos de investigación e implican la recolección y el análisis de datos cuantitativos y cualitativos, así como su integración y discusión conjunta, para realizar inferencias producto de toda la información recabada y lograr un mejor entendimiento del fenómeno bajo estudio" (p.546).

\section{Población seleccionada}

El estudio consideró entre sus fuentes de información en cada centro educativo: el director, el orientador, el profesor guía, los profesores de asignaturas regulares del currículum y los estudiantes de sétimo año.

Las instituciones educativas de secundaria seleccionadas pertenecen al circuito 05 de la Dirección Regional de Educación de Cartago. De las diecisiete instituciones de secundaria que componen el circuito educativo con distintas 
modalidades, se seleccionaron las tres que comparten la modalidad académica; a saber, el Colegio Francisca Carrazco, el Liceo Daniel Oduber y el Liceo Danilo Jiménez Veiga.

Según consta en las estadísticas de la Dirección Regional de Cartago del año 2010, el porcentaje de deserción a nivel de sétimo año, correspondiente a los colegios seleccionados fue el siguiente: Colegio Francisca Carrazco 12,97\%, Daniel Oduber 15,67\% y Liceo Danilo Jiménez Veiga $37,37 \%$.

\section{Selección de la muestra}

De acuerdo con la población de estudiantes de sétimo año de la educación general básica de las tres instituciones de secundaria seleccionadas, se realizó una selección de estudiantes mediante el tipo de muestra probabilística, Hernández y otros (2006) consideran que se obtiene cuando "todos los elementos de la población tienen la misma posibilidad de ser escogidos y se obtienen definiendo las características de la población y el tamaño de la muestra, y por medio de una selección aleatoria o mecánica de las unidades de análisis" (p. 241). En este sentido, se consideró el tamaño de la muestra mediante la fórmula de probabilidad

$$
\mathrm{n}=\frac{n_{0}}{1+\frac{n_{0}}{\mathrm{~N}}} \mathrm{y} n_{0}=\left(\frac{\mathrm{Z}}{\varepsilon}\right)^{2} \mathrm{~g} p \mathrm{~g} q
$$

atendiendo las siguientes condiciones: un nivel de confianza del $90 \%$ y asumiendo un error de cálculo del $10 \%$, donde se asigna la característica del porcentaje de deserción por institución del año 2010, de forma tal que $\mathrm{N}$ representa la población actual de sétimo año, $n_{0}$ es la muestra teórica calculada y $\mathrm{n}$ es la muestra real de la población asumida.

\section{CUADRO $\mathrm{N}^{\circ} 1$}

Muestra seleccionada del nivel de sétimo año de la Educación General Básica

\begin{tabular}{lccccc}
\multicolumn{1}{c}{ Institución Educativa } & Estudiantes & Docente Regular & Profesor Guía & Orientador & Director \\
Francisca Carrasco & 27 & 4 & 4 & 1 & 1 \\
Daniel Oduber Quirós & 42 & 6 & 3 & 2 & 1 \\
Danilo Jiménez Veiga & 33 & 4 & 2 & 1 & 1 \\
Total & 34 & 14 & 9 & 4 & 3 \\
\hline
\end{tabular}

Fuente: Elaboración propia, 2011.

Asimismo, de acuerdo con los objetivos propuestos en el estudio mediante una muestra no probabilística se seleccionaron del nivel de sétimo año de la Educación General Básica: docentes de asignaturas regulares del currículo, orientadores, profesores guías y directores de los colegios seleccionados. Según Hernández y otros (2006), en las muestras no probabilísticas "la elección de los elementos no depende de la probabilidad, sino de causas relacionadas con las características de la investigación o de quien hace la muestra" (p. 241).

\section{Instrumentos}

Para la recolección de los datos se utilizó la escala de actitud, el cuestionario semi-abierto y la entrevista semi-estructurada aplicados a los diferentes informantes: estudiantes de sétimo año, profesor guía, orientador, profesores de asignaturas regulares del currículum y directores. Para la validación del cuestionario dirigido a estudiantes, se escogieron cinco estudiantes de sétimo año en forma aleatoria. La validación de la escala Likert, y el cuestionario dirigido a profesor guía $\mathrm{y}$ orientador se valoraron a partir del criterio de 
expertos. Los datos fueron tabulados en tablas de Excel y se hizo una correlación según las categorías y subcategorías establecidas para el estudio.

\section{Resultados y análisis de los datos}

La utilización de diferentes instrumentos e informantes permitió triangulación de la información para lograr un análisis confiable respecto a la incidencia del clima institucional en la deserción de jóvenes en sétimo año de los colegios Daniel Oduber Quirós, Francisca Carrasco y Danilo Jiménez Veiga.

La presentación de los resultados se realiza mediante categorías y subcategorías. Entre las categorías planteadas en el estudio están, la cultura institucional, la mediación pedagógica y la gestión administrativa. Dentro de estos tres ejes temáticos directamente relacionados con los objetivos del estudio se redactaron indicadores que ordenan la recolección de los datos y su análisis. Entre las sub-categorías del estudio están la comunicación, la convivencia organizacional, las estrategias didácticas, el rol del estudiante, la infraestructura, y la implementación del plan estratégico institucional.

\section{Convivencia organizacional}

En cuanto a la sub categoría convivencia organizacional entendida como las acciones cotidianas que favorecen la relación de los estudiantes y el entorno educativo, es fundamental considerar los espacios recreativos en las instituciones educativas. De los 95 estudiantes encuestados en los tres colegios, el $82 \%$ opina que las instituciones cuentan con espacios recreativos que favorecen la convivencia estudiantil. El 27\% de los estudiantes del Francisca Carrasco difieren y expresan que no se cuenta con esos espacios.

Los espacios de acompañamiento fuera del aula y en espacios informales están ausentes en la mayoría de los casos. Se identifica un interés centrado principalmente en el rendimiento académico de los estudiantes. Ante la consulta abierta al profesor guía y orientador sobre la implementación de estrategias para la permanencia de los estudiantes de sétimo año, las principales estrategias en las que coinciden los tres colegios corresponden al análisis del rendimiento académico, seguimiento por parte del profesor guía y comunicación al hogar. Dos de los tres encuestados del Danilo Jiménez Veiga correspondiente a un $66 \%$, consideran que no se implementan estrategias y dos de los cinco encuestados que corresponde al 40\% del Daniel Oduber Quirós no responden la consulta.

El concepto de supervisión es entendido como sinónimo de vigilancia, en algunos casos por medio de cámaras, sin que los resultados de dichas observaciones se conviertan en insumos para ser abordados por los docentes guías $u$ orientadores de las instituciones.

En cuanto el fortalecimiento de las relaciones humanas, los resultados arrojan un interés particular por mantener una buena relación entre el personal docente y el administrativo, no se refleja un interés por considerar a los estudiantes o padres de familia en las estrategias de convivencia organizacional que plantea la institución.

\section{Comunicación}

La subcategoría comunicación, entendida como los espacios de expresión oral y escrita, permite el intercambio de información y conocimiento entre los diferentes actores. A pesar de que el $80 \%$ de los profesores guías y orientadores encuestados en cada uno de los colegios concuerda en que al menos una vez al mes conversan el profesor guía con el orientador para abordar situaciones de los estudiantes de sétimo año, los estudiantes coinciden en que no hay suficiente información en el tema de la resolución de conflictos. Ante la consulta sobre ¿cuáles aspectos se clarifican al inicio del curso? Los estudiantes encuestados en los tres colegios coinciden en que la evaluación de los aprendizajes de las diferentes asignaturas es el aspecto que se explica al inicio del curso. El aspecto sobre el que la mayoría de los encuestados expresa que recibe menos información al inicio del curso lectivo es en resolución de conflictos. 
A partir de los datos se identificó la ausencia de espacios de comunicación como un factor que incide en la deserción lo cual se convierte en un desafío para garantizar una convivencia organizacional efectiva en un marco de interacción y responsabilidad.

\section{Estrategias curriculares}

La recolección de los datos en la subcategoría "estrategias curriculares" hace referencia a las técnicas y actividades para el logro de los objetivos en la mediación pedagógica. Las estrategias curriculares definidas son un elemento curricular que involucra tanto al personal técnico como a los directores de las instituciones educativas. Ante la consulta abierta ¿de qué manera interviene el o la director(a) en los procesos de mediación pedagógica? Las respuestas varían; sin embargo, en su esencia se muestra que el director asigna esta función al coordinador académico, en algunos casos, abriendo espacios para trabajo en equipo entre colegas. Esto indudablemente reafirma la ausencia del director en acciones pedagógicas, tarea sustantiva de las instituciones educativas.

En cuanto al establecimiento de un perfil para docentes de sétimo año el $60 \%$ de los profesores guías y orientadores encuestados de las tres instituciones consideran que no existen lineamientos institucionales sobre el perfil del docente de sétimo año o no responden. A los directores de las tres instituciones se les preguntó sobre las características que se deben considerar en el perfil de un docente que imparta sétimo año y sobre las estrategias institucionales para abordar algunos de los aspectos mencionados. En todos los casos, mencionan elementos fundamentales como la experiencia, vocación y estabilidad laboral; sin embargo, el seguimiento en cuanto a cómo estas características influyen en el interés de los jóvenes de sétimo año, no es un aspecto al que se le brinde seguimiento a lo interno de la institución educativa.

\section{Rol del estudiante}

La subcategoría "rol del estudiante" lo considera como un sujeto activo con deberes y responsabilidades en proceso de enseñanza y aprendizaje. En cuanto al cumplimiento de los estudiantes con los deberes y responsabilidades; así como las razones que los motivan a estudiar, se infiere que el profesor brinda apoyo y facilita la mediación pedagógica; no obstante, los estudiantes tienen necesidades particulares de las cuales el personal docente y administrativo no está enterado.

Ante la consulta semi-abierta dirigida a los estudiantes de sétimo año, sobre las razones por las cuáles estudian en la educación secundaria, seleccionan como principal razón me gusta estudiar, y la menos elegida corresponde a incentivo económico. A partir de la respuesta, se puede inferir que los estudiantes asisten al colegio principalmente por tener una actitud positiva hacia la educación y no necesariamente por una obligación o incentivo para que permanezcan en las aulas.

Ante esta situación es indispensable que el profesor brinde apoyo y desarrolle la mediación pedagógica tomando como referencia no solamente los contenidos curriculares establecidos en el plan de estudios, sino también las motivaciones, prioridades e intereses de los jóvenes.

\section{Infraestructura}

La infraestructura escolar hace referencia a los ambientes educativos propicios para los procesos de enseñanza y aprendizaje, los cuales tienen relación con el confort humano y por ende el desempeño de los estudiantes. En este sentido, la subcategoría infraestructura indaga sobre las aulas, los pupitres y los servicios sanitarios en los tres colegios.

El 98\% de los estudiantes encuestados del Daniel Oduber opina que las aulas tienen las 
condiciones óptimas para el desarrollo de la lección. Igualmente, en el Francisca Carrasco el 80\% considera que se cuenta con las aulas en condiciones óptimas. En menor grado, los estudiantes del Danilo Jiménez Veiga, un $60 \%$, coincide en que se cuenta con condiciones de infraestructura para el desarrollo de las lecciones, un 34\% opina que no se cuenta en la institución con las aulas en condiciones óptimas. A partir de los datos recolectados, se puede inferir que en las tres instituciones consultadas se generan diferencias de opinión entre los informantes con respecto a la infraestructura. Para algunos, las condiciones son óptimas, pero para otros deben mejorar; sin embargo, en términos generales, los estudiantes y docentes muestran satisfacción respecto a las instalaciones de los colegios.

\section{Plan Estratégico Institucional}

Las tendencias educativas han transformado las prácticas administrativas en la gestión de los centros educativos. Como resultado, el MEP ha incluido entre los lineamientos la implementación del PEI. Este tiene un carácter instrumental que permite orientar el recurso humano y financiero en las propuestas educativas.

Los directores de las tres instituciones fueron consultados sobre las ventajas y desventajas del PEI. Los tres directores coinciden en que este es un instrumento que da el norte a la institución. Igualmente coinciden que la construcción del PEI involucra personal técnico, administrativo y juntas administrativas. Sin embargo, al consultar al personal docente se vislumbra que la construcción pedagógica no es un proceso conocido por todo el personal docente. En la consulta cerrada el $30 \%$ de los profesores guía y orientadores encuestados opinan que sí participan en la construcción del PEI. Sin embargo, en la sección abierta del cuestionario pareciera que los profesores no tienen claro que es el PEI, terminología que según los lineamientos del MEP es parte fundamental en la planificación institucional. El otro $70 \%$ no participa o no responde.

En este sentido, se muestra ausencia de prácticas que permitan procesos participativos en la construcción y reconstrucción de propuestas curriculares en el nivel institucional. En realidad, el estudio encuentra que se utiliza como principal medio de comunicación para aspectos de carácter técnico y administrativo el envío de oficios, los cuales son de acatamiento obligatorio y siempre con un carácter informativo y no de consenso entre las partes.

\section{Conclusiones}

La convivencia entre docentes y estudiantes en actividades de recreación y esparcimiento para compartir y desarrollar vínculos socio afectivos entre pares, personal docente y administrativo no son iniciativas propias de las instituciones; sino más bien, respuesta a lineamientos del MEP.

El tipo de comunicación que predomina entre los participantes del estudio es la formal, involucra el uso del cuaderno de comunicaciones, protocolos institucionales de cómo proceder en temas como las ausencias injustificadas.

En lo que respecta al trabajo en equipo entre el personal docente y administrativo, este se asocia con la delegación de roles a algunos funcionarios, lo que implica escasa oportunidad de construcción y acompañamiento de propuestas integrales.

El plan estratégico institucional es un aspecto de la planificación estratégica de centro educativo poco reconocido por los miembros de la comunidad educativa encuestada.

Las tres instituciones procuran la ejecución de estrategias didácticas que favorezcan la permanencia de los estudiantes de sétimo año; pero no hay referencia de parte de los profesores de sétimo año en los procesos de trabajo colaborativo. En algunos casos se menciona al coordinador académico con una función fiscalizadora y no de consenso entre los colegas de las áreas comunes.

En cuanto a la infraestructura las opiniones son divididas entre los encuestados; sin embargo, la mayoría algunos coinciden en que las condiciones de infraestructura y mobiliario son buenas.

Los estudiantes encuestados expresan la falta de acompañamiento en el tema de resolución de conflictos, el abordaje de resolución de 
conflictos implica un proceso continuo con diversas temáticas y no únicamente actividades aisladas llevadas a cabo por algunos miembros de la comunidad educativa.

\section{Recomendaciones}

Realizar acciones institucionales administrativas, pedagógicas y técnicas enfocadas al desarrollo integral del ser humano, con el propósito de mejorar el rendimiento escolar y la permanencia de los jóvenes.

Reconocer a lo interno de la institución que el abordaje pedagógico demanda compromiso e identificación del personal docente y del director, por lo que se deben propiciar espacios para debates, reflexiones y decisiones que favorezcan la percepción de la comunidad educativa y los resultados académicos de los estudiantes.

Incorporar en la mediación pedagógica espacios para que el estudiante comprenda lo que está construyendo académicamente y desarrolle las habilidades y destrezas fundamentales para abordar aspectos tales como la resolución de conflictos.

Velar por el estado y condiciones óptimas de la infraestructura escolar, pero aún más importante, será hacer de estas el espacio donde los jóvenes desarrollen los procesos de enseñanza y aprendizaje desde una perspectiva integral del ser humano.

Favorecer espacios de convivencia organizacional en el que se apoye el liderazgo de los diferentes miembros de la comunidad educativa, con el objetivo de potenciar las fortalezas del personal y fomentar una cultura institucional participativa.

Elaborar un protocolo institucional de inducción y acompañamiento para los estudiantes de sétimo año con la participación activa de profesores, administrativos y padres de familia. El protocolo debe incluir la resolución de necesidades sustentadas en la realidad y una fundamentación teórico-conceptual sobre la importancia de implementar estrategias diferenciadas con los estudiantes de sétimo año.

Elaborar el PEI con la representación de todas las partes y promover a lo interno del equipo de trabajo espacios de socialización y de seguimiento a las acciones establecidas. Es fundamental la planificación de programas de intervención dirigidos al fortalecimiento de las relaciones humanas en los que se favorezca la participación activa del personal docente, una actitud de compromiso con la planificación institucional y una visión del estudiante como ser humano integral.

\section{Bibliografía}

Constitución Política de Costa Rica. (1979). San José, Costa Rica.

Consejo Superior de Educación. (2008). El Centro Educativo de calidad como Eje de la Educación. San José Costa Rica.

Gibson, J. y Colbs. (2001). Las organizaciones: Comportamiento Estructura-Procesos.

México: Mc. Graw. Hill.

Hernández, R. Fernández C. y Baptista P. (2006). Metodología de la investigación organizacional. 4a. ed. México: McGraw-Hill.

Jabif, L. (2009). Formación en competencias para la gestión escolar en contextos de pobreza. Ministerio de Educación Pública: San José Costa Rica.

Letjman, S. (1996). La conducción de una institución educativa, en organizar y conducir la escuela: Reflexiones de cinco directores y un asesor. Argentina: Editorial Paidós.

Ministerio de Educación Pública. (2010). Líneas estratégicas MEP 2010-2014.San José, Costa Rica. 
- 